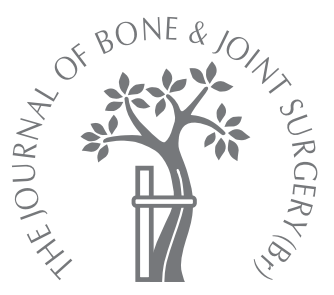

\title{
Free vascularised iliac bone graft for recalcitrant avascular nonunion of the scaphoid
}

\author{
R. Arora, \\ M. Lutz, \\ R. Zimmermann, \\ D. Krappinger, \\ C. Niederwanger, \\ M. Gabl \\ From Medical \\ University Innsbruck, \\ Innsbruck, Austria
}

\begin{abstract}
We report the use of a free vascularised iliac bone graft in the treatment of 21 patients (19 men and 2 women) with an avascular nonunion of the scaphoid in which conventional bone grafting had previously failed. The mean age of the patients was 32 years (23 to 46$)$ and the dominant wrist was affected in 14 . The mean interval from fracture to the vascularised bone grafting was 39 months (9 to 62). Pre-operative MRI showed no contrast enhancement in the proximal fragment in any patient. Fracture union was assessed radiologically or with CT scans if the radiological appearances were inconclusive.

At a mean follow-up of 5.6 years ( 2 to 11$)$ union was obtained in 16 patients. The remaining five patients with a persistent nonunion continued to experience pain, reduced grip strength and limited range of wrist movement. In the successfully treated patients the grip strength and range of movement did not recover to match the uninjured side.

Prevention of progressive carpal collapse, the absence of donor site morbidity, good subjective results and pain relief, justifies this procedure in the treatment of recalcitrant nonunion of the scaphoid.
\end{abstract}

Scaphoid fractures are sometimes unrecognised, but even when primarily recognised and treated appropriately, nonunion may occur in $5 \%$ to $15 \% .^{1}$ Pseudarthrosis of a scaphoid fracture usually results from a combination of a long-standing nonunion, previous failed surgery, avascularity of the proximal fragment or in certain fractures of the proximal third of the scaphoid. ${ }^{2}$ Observations on the natural history of scaphoid nonunion reveal that osteoarthritis is time dependent and may become progressively worse in association with long-standing nonunion. Therefore, most hand surgeons would recommend surgical treatment of symptomatic nonunion. ${ }^{3}$

In scaphoid fractures with a long-standing nonunion and in those where previous surgery has failed, the results of conventional bone grafting are generally poor, ${ }^{4}$ although good results have been reported following rib graft arthroplasty. ${ }^{5}$ Recently vascularised bone grafts have been recommended for persisting scaphoid nonunions after failed earlier surgery and for nonunions with evidence of avascular necrosis (AVN). ${ }^{6}$ A vascularised periosteal graft may also give good results. ${ }^{7}$ We evaluated the use of a free vascularised iliac crest bone graft and internal fixation with a Kirschner $(\mathrm{K})$-wire to treat patients who had recalcitrant scaphoid nonunions with evidence of AVN after at least two previous failed operations. In particular, we examined the rate of union and the functional outcome from this extensive procedure.

\section{Patients and Methods}

Patients were selected for this study from a population of 94 patients undergoing reconstructive surgery for nonunion of scaphoid fractures treated at our institution between 1994 and 2003. Inclusion criteria and the indication for free vascularised iliac crest bone grafting were limited to patients with recalcitrant nonunion. This was defined as persistence of a fracture gap at least nine months after a second attempt at non-vascularised bone grafting, with associated resorption of bone and cystic changes at the fracture site, ${ }^{8}$ which itself followed failed initial internal screw fixation and failure of subsequent attempted conventional bone grafting. In all, patients were identified who had two previous failed surgical attempts at achieving union. Patients with advanced collapse were excluded, as were patients who had established radioscaphoid arthritis for which a four-corner arthrodesis ${ }^{9}$ was performed $(n=9)$, and patients with arthritis throughout the carpus who had an arthrodesis of the wrist $(n=7)$.

The remaining 26 patients who had no signs of arthritis were treated by free vascularised iliac bone grafting and internal fixation with a 


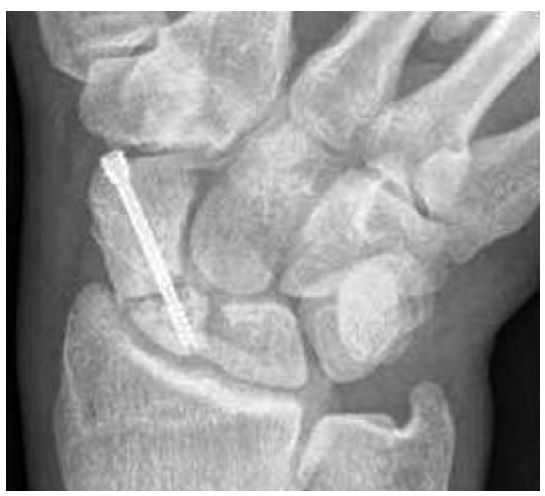

Fig. 1a

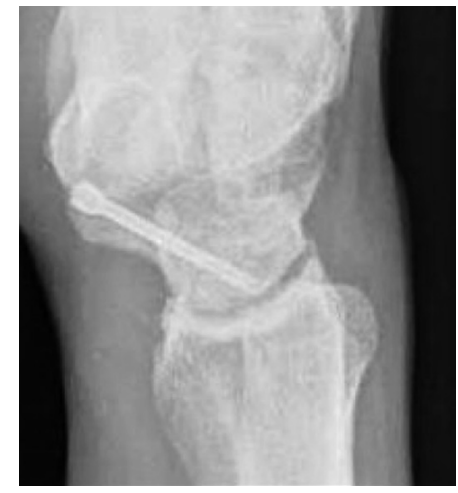

Fig. 1b

Radiographs of a 26-year-old man showing a proximal pole nonunion ten months after screw fixation. (a) The posteroanterior view shows a central lucency, consistent with avascular necrosis. (b) The lateral view, showing no carpal malalignment.

K-wire. Of these, three patients did not attend the final follow-up and two others were excluded because of incomplete documentation in relation to the previous procedures, leaving 21 in the final analysis. This group comprised 19 men and two women, with a mean age at surgery of 32 years (23 to 46); the dominant wrist was affected in 14 . A total of 13 patients were employed in manual labour, five had office-based jobs and three were unemployed. The fractures were sustained in road traffic accidents in eight patients, nine were related to a sporting injury and another four due to a fall. Of the 21 patients nine were smokers and were advised to cease smoking to improve the likelihood of a successful outcome from this treatment.

All patients had been treated initially by percutaneous distal screw fixation (Fig. 1). The nature of the failed secondary bone graft procedures varied, with six patients having removal of the screw and a conventional Russe bone graft $^{10}$ (Fig. 2), four undergoing interpositional anterior wedge grafting and repeated internal fixation, and 11 undergoing open reduction with cancellous bone graft with repeated internal compression screw fixation. The nonunion was located at the waist of the scaphoid in 15 and in the proximal third in six patients. Seven of the 15 patients with a fracture of the middle third of the scaphoid presented with a humpback deformity with accompanying dorsal intercalated segment instability (DISI). ${ }^{11}$ Pre-operative MRI showed no contrast enhancement in the proximal scaphoid in any patient indicating avascularity. ${ }^{12}$ This was also confirmed in all patients at operation by the absence of punctuate bleeding from the proximal fragment. ${ }^{13}$ The mean interval between injury and vascularised bone grafting was 39 months (9 to 62).

Prior to surgery all patients complained of wrist pain, reduced grip strength and/or reduced range of wrist movement.

Functional assessment. Functional outcome was assessed by an orthopaedic specialist physiotherapist who was not blinded to the method of treatment, as the palmar-located scars could easily be seen, and included pre- and post-operative measurement of active range of wrist movement using a goniometer. Grip power was measured with a Jamer dynamometer (Therapeutic Equipment, Clifton, New Jersey). Final clinical results were compared to the pre-operative measurements and the uninjured contralateral side. Wrist pain was evaluated using a visual analogue scale (VAS $0=$ no pain, VAS $100=$ severe pain). Functional subjective outcome was measured with the Disability of the Arm, Shoulder, and Hand (DASH) Questionnaire. ${ }^{14}$ This has a 0 to 100 point scale where 0 represents the best result. ${ }^{14}$ In addition, at final review the Patient-Rated Wrist Evaluation Score ${ }^{15}$ was also established. In this system there is a scale of 0 to 150 points, where there are no points for an asymptomatic wrist. Subjective and objective data were summarised in the modified Green and O'Brien Score, ${ }^{15}$ where 100 points were awarded for the best and no points for the worst outcome ( $>90$, excellent; 75 to 89 , good; 60 to 74 , fair; <60, poor).

Radiological assessment. Standard posteroanterior (PA), lateral and oblique radiographs of the wrist were taken. Assessment was performed by a reviewer $(\mathrm{CN})$ who was not a member of the surgical team and who was blinded to the functional outcome but not to the method of treatment. The radiographs at the final follow-up were examined for signs of osteoarthritis using the staging system of Watson and Ryu. ${ }^{16}$ The pre- and post-operative radiographs were further assessed for the scapholunate angle and the radiolunate angle. Changes in the scapholunate angle were evaluated to assess palmar rotation of the scaphoid. The presence of DISI was assessed by changes in the radiolunate angle on lateral radiographs. ${ }^{17}$ The uninjured contralateral wrist served as the control for these measurements. Fracture union was defined according to the criteria described by Dias $^{18}$ and was assessed at final follow-up from radiographs or with CT scans if the radiological appearances were inconclusive. 


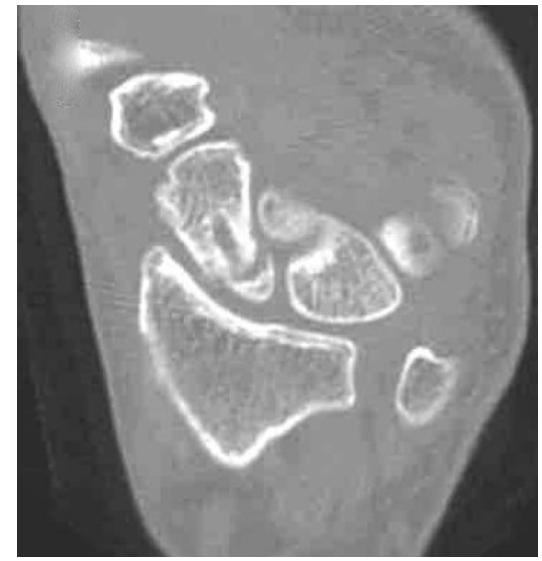

Fig. 2

Coronal CT scan of the same patient as in Figure 1 after removal of the initial implanted screw and a failed conventional Russe bone graft 19 months after initial screw fixation.

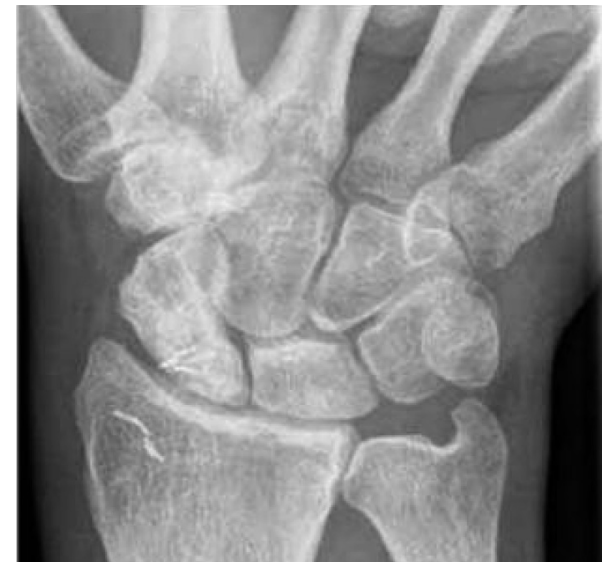

Fig. 3

Posteroanterior radiograph of the patient shown in Figure 1 obtained at final follow-up eight years post-operatively. There is complete healing and proximal pole revascularisation with no evidence of osteoarthritis or carpal collapse.
Statistical analysis. The software package SPSS for Windows v 16.0 (SPSS Inc., Chicago, Illinois) was used for statistical analysis. A paired Student's $t$-test was performed and numerical data are expressed as mean and SD or range. A p-value of $<0.05$ was considered statistically significant. Surgical technique and post-operative management. The operation was performed under general anaesthesia with tourniquet control and loupe magnification. Patients were placed in a supine position with the arm abducted at $90^{\circ}$. Debridement of the scaphoid and harvest of the vascularised bone graft from the iliac crest were performed simultaneously by two separate teams. The deep circumflex iliac vascular bundle with its fascial layer was identified through an incision parallel to the inguinal ligament and traced laterally. The tiny vascular branches running to the iliac crest were identified and, according to the site of their penetration into the bone, a tricortical bone graft was elevated. Vascularity of the graft was confirmed by its bleeding. The scaphoid was then simultaneously inspected through the previous volar incision, which was extended distally to the tubercle of the scaphoid and proximally over the flexor carpi radialis tendon for $2 \mathrm{~cm}$. The volar wrist capsule with the long radiolunate and radioscaphocapitate ligaments was sharply divided, exposing the scaphoid. The fibrous nonunion was debrided and the necrotic bone of the proximal part was excised under image intensifier control, leaving the entire shell of cartilage with a thin layer of subchondral bone. Following shaping of the iliac bone graft with its cortical surface, including the periosteum and penetrating vessels positioned on the palmar aspect, the graft was interposed between the two fragments of the scaphoid. In cases with a humpback deformity the graft was shaped as a trapezoidal wedge to correct this. In order to prevent displacement, the graft was fixed with a $1 \mathrm{~mm}$ diameter $\mathrm{K}$-wire. The vessels were then anastomosed end-to-side for the radial artery and end-to-end for its venae comitantes. The period of immobilisation after surgery followed a protocol with a below-elbow cast retained for 12 weeks, at which stage the K-wire was removed. This was followed by passive and assisted active wrist exercises.

\section{Results}

The mean follow-up after the vascularised bone grafting was 5.6 years (2 to 11), at which time union was confirmed radiologically in 16 of the 21 patients (Fig. 3).

Patients with union at final follow-up. The results for range of movement and grip strength are shown in Table I. The mean time to union after vascularised grafting was 17 weeks (14 to 20) for nonunions of the waist, and 21 weeks (18 to 23) for nonunions of the proximal pole. In the 16 patients who achieved union, wrist extension, radial deviation of the wrist, and grip strength increased after surgery but was accompanied by a reduction in active flexion and ulnar deviation. Only the difference in the pre- and post-operative grip strength were statistically significant $(\mathrm{p}=0.03)$. At final follow-up a comparison with the uninjured wrist demonstrated the mean flexion-to-extension arc, the mean ulnar radial deviation arc and the mean grip strength were restored to $68 \%, 73 \%$ and $81 \%$ of uninjured side, respectively. There was no pre- or postoperative limitation of pronation or supination.

The mean pain level measured by VAS decreased from 16.0 points ( 4 to 40 ) before surgery to 2.6 points ( 0 to 9 ) at the final follow-up $(\mathrm{p}=0.03)$. Three patients had to change their occupation because of prolonged pain during attempts to return to their pre-injury employment.

The mean DASH score at final follow-up was 14.8 points (SD 10.7) and the mean patient related wrist evaluation 
Table I. Summary of mean and SD of the clinical and radiological results with comparison between pre-operative and final follow-up. The results of the final follow-up and uninjured contralateral side have been compared and the $p$-value presented

\begin{tabular}{|c|c|c|c|c|}
\hline & \multicolumn{4}{|c|}{ Before surgery (SD) Final follow-up (SD) p-value Uninjured contralateral side (SD) } \\
\hline \multicolumn{5}{|l|}{ All patients $(n=21)$} \\
\hline Arc of extension $\left({ }^{\circ}\right)$ & $44(16)$ & $45(13)$ & 0.79 & $67(7)$ \\
\hline Arc of flexion $\left({ }^{\circ}\right)$ & $46(9)$ & $36(9)$ & 0.08 & $64(7)$ \\
\hline Arc of ulnar deviation $\left({ }^{\circ}\right)$ & $28(5)$ & $24(9)$ & 0.39 & $41(6)$ \\
\hline Arc of radial deviation $\left({ }^{\circ}\right)$ & $19(9)$ & $17(8)$ & 0.86 & $28(8)$ \\
\hline Grip strength (kg) & $25(4)$ & $34(13)$ & 0.18 & $50(11)$ \\
\hline Scapholunate angle $\left({ }^{\circ}\right)$ & $51(6)$ & $49(5)$ & 0.68 & $46(7)$ \\
\hline Radiolunate angle $\left({ }^{\circ}\right)$ & $18(3)$ & $11(5)$ & 0.23 & $7(4)$ \\
\hline \multicolumn{5}{|l|}{ Patients with union $(n=16)$} \\
\hline Arc of extension $\left({ }^{\circ}\right)$ & $44(17)$ & $49(11)$ & 0.57 & $68(7)$ \\
\hline Arc of flexion $\left({ }^{\circ}\right)$ & $46(8)$ & $38(7)$ & 0.10 & $64(7)$ \\
\hline Arc of ulnar deviation $\left({ }^{\circ}\right)$ & $28(5)$ & $25(10)$ & 0.50 & $40(6)$ \\
\hline Arc of radial deviation $\left({ }^{\circ}\right)$ & $17(9)$ & $18(8)$ & 0.87 & $28(7)$ \\
\hline Grip strength $(\mathrm{kg})$ & $25(3)$ & 39 (9) & 0.03 & $51(11)$ \\
\hline Scapholunate angle $\left({ }^{\circ}\right)$ & $51(6)$ & 47 (3) & 0.23 & $45(7)$ \\
\hline Radiolunate angle $\left({ }^{\circ}\right)$ & $17(3)$ & $10(4)$ & 0.18 & $7(3)$ \\
\hline \multicolumn{5}{|c|}{ Patients with persisting nonunion $(n=5)$} \\
\hline Arc of extension $\left({ }^{\circ}\right)$ & $43(15)$ & $32(9)$ & 0.04 & $66(6)$ \\
\hline Arc of flexion $\left({ }^{\circ}\right)$ & $46(10)$ & $31(8)$ & 0.02 & $65(7)$ \\
\hline Arc of ulnar deviation $\left({ }^{\circ}\right)$ & $28(6)$ & $22(8)$ & 0.04 & $41(6)$ \\
\hline Arc of radial deviation $\left({ }^{\circ}\right)$ & $18(9)$ & $12(6)$ & 0.04 & $27(8)$ \\
\hline Grip strength (kg) & $26(5)$ & $20(9)$ & 0.02 & 49 (9) \\
\hline Scapholunate angle $\left(^{\circ}\right)$ & $50(5)$ & $56(3)$ & 0.31 & $46(6)$ \\
\hline Radiolunate angle $\left({ }^{\circ}\right)$ & $18(3)$ & $15(4)$ & 0.27 & $6(4)$ \\
\hline
\end{tabular}

score was 20 points (SD 9.8). Of the 16 patients with union of the scaphoid the Green and O'Brien score showed eight excellent, five good, two fair and one poor result.

At final follow-up, 11 of the 16 patients with union showed no signs of osteoarthritis and five showed stage I arthritis. In this group the scapholunate angle was a mean of $47^{\circ}(96 \%)$ compared with the uninjured side and the radiolunate angle was partly restored, being a mean of $10^{\circ}$ $(70 \%)$ compared with the uninjured side. These changes of the radiological parameters did not reach statistical significance (Table I). In all patients with humpback deformity and associated DISI, this deformity had been improved post-operatively and did not deteriorate with follow-up.

Patients with persisting nonunion at final follow-up. The results of range of movement and grip strength are shown in Table I. For this group of five patients the mean time between injury and vascularised bone grafting was 52 months (39 to 62) and the wrist extension, flexion, radial and ulnar deviation and the grip strength all decreased statistically significantly after surgery. At final follow-up the mean flexion-extension and ulnar to radial wrist deviation arc and the grip strength were $48 \%, 50 \%$ and $41 \%$, respectively. There was no pre- or post-operative limitation of pronation or supination.

The mean pain level measured by VAS increased from 15.6 points (6 to 48 ) before surgery to 31.2 points (12 to $78)$ at the final follow-up $(\mathrm{p}=0.02)$.
The mean DASH score at final follow-up was 40.2 points (SD 8.9) and the mean patient rated wrist evaluation score was 54.2 points (SD 16.8). The Green and O'Brien score showed poor results with a mean of 86 points (59 to 100) for all patients.

At final follow-up all patients had radiological evidence of osteoarthritis. In three patients this was stage 2 and in two it was stage 3 . The mean scapholunate angle and mean radiolunate angle were $92 \%$ and $40 \%$, respectively, of those shown in the uninjured wrist (Table I). Each of these patients had to change their occupation owing to symptoms from the wrist. None wished further treatment.

Complications. Minor complications occurred in four patients. These included one prominent K-wire, which was noticeable under the skin and caused pain. It was removed eight weeks after operation without any signs of secondary graft displacement. Three patients had a superficial infection which resolved with dry dressings. There was no donor site morbidity due to infection, hernia formation or injury to the lateral cutaneous nerve of the thigh, although all patients reported pain at the donor site during the first four weeks after the surgery. At the final follow-up this pain had resolved entirely in all patients.

\section{Discussion}

Contemporary literature indicates that, besides achieving union, restoration of the anatomical scaphoid length and 
carpal alignment is correlated with better functional results, whereas untreated, displaced scaphoid nonunions will lead to secondary arthritis changes and carpal collapse. ${ }^{19}$ Therefore, in patients with no evidence of degenerative changes, achieving union of long-standing scaphoid nonunion should be attempted. The Russe inlay bone graft technique remains standard treatment for scaphoid nonunion. ${ }^{20}$ However, although a union rate of $92 \%$ has been reported, ${ }^{21}$ it is unlikely to be successful when previous conventional grafting has failed. Carrozella et $\mathrm{al}^{21}$ achieved union in only $60 \%$ using a non-vascularised bone graft after failure of a previous conventional bone graft. Boyer, von Schroeder and Axelrod ${ }^{22}$ suggested that a history of previous surgery was one of the main causes of an unsatisfactory result after a second attempted grafting.

We assume that each operation on the scaphoid damages the surrounding soft tissues, and that any further excision of fibrous tissue at the nonunion site worsens the scaphoid blood supply. It follows that most scaphoid fractures that fail to heal with conventional bone grafting probably have impaired vascularity. ${ }^{23}$ Vascularised bone grafting is currently accepted in treating nonunion of the scaphoid with an avascular proximal pole and following failed previous surgery. ${ }^{24,25}$ It has been suggested that vascularised bone grafts are more effective in unfavourable, scarred and irradiated recipient sites such as occur with a recurrent pseudoarthrosis. ${ }^{26}$ Whereas non-vascularised bone grafts heal by creeping substitution, involving gradual resorption of the graft followed by callus formation, vascularised bone grafts heal without extensive resorption, callus formation or remodelling. Therefore, bone viability and mechanical strength are preserved during bone healing. By providing osteo-inductive and conductive stimuli, union appears to be faster in vascularised bone grafts. ${ }^{27}$

Such grafts can have pedicles based on the arterial blood supply from the recurrent palmar branch of the radial artery, ${ }^{28}$ the second dorsal metacarpal artery, ${ }^{29}$ the pronator quadratus pedicles artery ${ }^{30}$ and the ulnar artery. ${ }^{31}$ Generally a short arc of graft rotation and no continuity of the small muscular bundle make grafts harvested from the palmar aspect of the distal radius potentially problematical when pedicled on the pronator quadratus and the palmar radiocarpal artery. ${ }^{27}$ The disadvantages of vascularised bone grafts based on the ulnar artery are the risk of fracturing the ulna and the need to reconstruct the sacrificed ulnar artery using a vein graft.

Recently, vascularised bone graft from the dorsal aspect of the radius with the pedicle based on a 1,2 intercompartmental supraretinacular (1, 2 ICSRA) branch of the radial artery has become the most commonly used vascularised bone graft in the treatment of proximal pole nonunions with avascular necrosis when conventional bone grafting has failed. ${ }^{32}$

Boyer et $\mathrm{al}^{22}$ reported union in six of ten patients treated with the 1, 2 ICSRA-based vascularised graft. The four persistent nonunions had all been treated previously with nonvascularised bone grafts.
In contrast, Straw et $\mathrm{al}^{25}$ reported a union rate of $27 \%$ after the use of vascularised grafts based on the 1,2 ICSRA, with 16 of the 22 nonunions noted to have an avascular proximal pole, of which only two went on to union. In total, five patients had undergone previous failed conventional bone grafting. The authors suggested that 1,2 ICSRA-based vascularised bone grafts did not improve the rate of union in scaphoid nonunions with an avascular proximal pole.

Tambe et $\mathrm{al}^{33}$ described 11 patients treated with vascularised bone grafts from the dorsum of the distal radius. In this series all patients had a minimum of two previous failed operations with conventional bone grafting. Punctuate bleeding from the proximal pole was absent in all patients. In two patients a pedicle based on a 1, 2 ICSRA was used and in the remainder various branches of extracompartmental artery were used. At final follow-up the nonunion in six of the 11 patients remained ununited, for which additional corticocancellous bone grafting from the iliac crest or the distal radius was used. Correction of any humpback deformity from a single dorsal approach was not possible, and the overall union rate was $55 \%$ (6 of 11) patients.

It has been suggested that scaphoid nonunions with substantial bone loss and a humpback deformity should not be expected to heal with a vascularised bone graft based on the 1, 2 ICSRA, as the bone grafts have to be rotated palmarly and inserted from the volar side of the nonunion, which may result in kinking of the pedicle during insertion.

With our procedure we obtained union in 16 of 21 patients with partial correction of any palmar rotation of the scaphoid and DISI deformity. Nevertheless, at final follow-up five of 16 patients with bone union had developed stage 1 arthritis, which remained limited to the radioscaphoid joint. The advantage of free vascularised iliac crest bone graft was the ease of shaping it to fill the defect with no interference with the attached vessels. The dense structured cancellous bone graft with its strong cortical bone was placed to the palmar aspect of the scaphoid, where the maximal axial load is applied. ${ }^{34}$ The larger cortical portions from the iliac crest stabilised the graft during positioning and allowed restoration of scaphoid height and correction of DISI in cases of humpback deformity.

It has been recognised that, compared with the distal radial graft, the iliac crest graft is composed of dense material which is mechanically superior. Bone volume and bone turnover of the distal radius is lower, the trabeculae are thinner, and the cortices are only half as thick as the iliac crest. ${ }^{35}$

It is interesting that even where union was achieved the post-operative range of wrist movement did not improve significantly relative to the pre-operative measurements and remained less than that of the uninjured side. These findings mirror those of other studies. ${ }^{36}$ The overall objective and subjective patient satisfaction, as assessed by the DASH score and patient rated wrist evaluation score, was a mean of 20 and 14.8 points, respectively. The Green and O'Brien score showed $81 \%$ excellent and good results. 
Most studies on vascularised bone grafting have limited their outcome assessment to bone healing, ${ }^{37}$ with only a few reporting an excellent or good outcome in $64 \%{ }^{38}$ to $77 \%{ }^{25}$ of their patients.

Failure to obtain union in every patient might be partially attributable to differences in location of the scaphoid nonunion, tobacco use and the duration of the nonunion. ${ }^{39}$ Three of five patients with persistent nonunion had the fracture located in the proximal third of the scaphoid. All the patients in whom the nonunion failed to heal were smokers. The failed group also had a larger mean period between injury and vascularised bone grafting. Our results confirmed the findings from previous studies stating that the site of injury, tobacco use and age of the nonunion are important determinants of outcome. ${ }^{39}$

As it is accepted that patients with a persistent scaphoid nonunion will develop progressive, degenerative changes with time, ${ }^{3}$ and long-standing carpal malalignment causes restriction of wrist movement, ${ }^{36}$ we believe that the deterioration of radiological and functional results in patients with persistent nonunion were due to natural progression.

On the basis of our findings, patients with recalcitrant avascular scaphoid nonunion should be informed preoperatively that vascularised iliac bone grafting will reduce pain and improve grip strength, but will not to match the uninjured contralateral side. Additionally, they should be aware that the range of wrist movement will not return to the pre-injury level. We do not recommend this procedure for patients who are unable to stop smoking.

We recognise the limitations of our study due to its retrospective design and the absence of a corresponding cohort of patients treated by a non-vascularised graft for comparison. Nevertheless, the use of a free vascularised iliac crest bone graft in patients with recalcitrant avascular scaphoid nonunion after previous failed conventional bone graft procedures resulted in union in just over threequarters of patients.

No benefits in any form have been received or will be received from a commer cial party related directly or indirectly to the subject of this article.

\section{References}

1. Cooney WP, Linscheid RL, Dobyns JH. Scaphoid fractures: problems associated with nonunion and avascular necrosis. Orthop Clin North Am 1984;15:381-91.

2. Osterman AL, Mikulics M. Scaphoid nonunion. Hand Clin 1988;4:437-55

3. Mack GR, Bosse MJ, Gelberman RH, Yu E. The natural history of scaphoid nonunion. J Bone Joint Surg [Am] 1984;66-A:504-9

4. Green DP. The effect of avascular necrosis on Russe bone grafting for scaphoid nonunion. J Hand Surg [Am] 1985;10:597-605.

5. Veitch S, Blake SM, David H. Proximal scaphoid rib graft arthroplasty. J Bone Joint Surg [Br]2007;89-B:196-201.

6. Moran SL, Shin AY. Vascularized bone grafting for the treatment of carpal pathology. Orthop Clin North Am 2007;38:73-85.

7. Thompson NW, Kappoor A, Thomas J, Hayton MJ. The use of a vascularised periosteal patch onlay graft in the management of nonunion of the proximal scaphoid. J Bone Joint Surg [Br] 2008;90-B:1597-601.

8. Simonian PT, Trumble TE. Scaphoid nonunion. J Am Acad Orthop Surg 1994;2:185-91.

9. Kendall CB, Brown TR, Millon SJ, et al. Results of four-corner arthrodesis using dorsal circular plate fixation. J Hand Surg [Am]2005;30:903-7.
10. Bynum EB, Culp RW, Bonatus TJ, Alexander CE, McCarroll HR. Repeat Russe bone grafting after failed bone graft surgery for scaphoid non-union. J Hand Surg $[\mathrm{Br}]$ 1995;20:373-8.

11. Tomaino MM, King J, Pizillo M. Correction of lunate malalignment when bone grafting scaphoid nonunion with humpback deformity: rationale and results of a technique revisited. J Hand Surg [Am] 2000;25:322-9.

12. Cerexal L, Abascal F, Canga A, et al. Usefulness of gadolinium-enhanced MR imaging in the evaluation of the vascularity of scaphoid nonunions. AJR Am J Roentgenol 2000;174:141-9.

13. MacDermid JC. Development of a scale for patient rating of wrist pain and disability. J Hand Ther 1996;9:178-83.

14. MacDermid JC, Richards RS, Donner A, Bellamy N, Roth JH. Responsiveness of the short form-36, disability of the arm, shoulder, and hand questionnaire, patientrated wrist evaluation, and physical impairment measurements in evaluating recovery after distal radius fracture. J Hand Surg [Am]2000;25:250-330-40.

15. Green DP, O'Brien ET. Open reduction of carpal dislocations: indications and operative techniques. J Hand Surg [Am] 1978;3:250-65.

16. Watson HK, Ryu J. Evolution of arthritis of the wrist. Clin Orthop 1986;202:57-67.

17. Linscheid RL, Dobyns JH, Beabout JW, Bryan RS. Traumatic instability of the wrist: diagnosis, classification, and pathomechanics. J Bone Joint Surg [Am]2002;84A:142.

18. Dias JJ. Definition of union after acute fracture and surgery for fracture nonunion of the scaphoid. J Hand Surg [Br] 2001;26:321-5

19. Herbert TJ. Natural history of scaphoid nonunion: a critical analysis. J Hand Surg [Am] 1994;19:155-6.

20. Barton NJ. Experience with scaphoid grafting. J Hand Surg [Br] 1997;22:153-60.

21. Carrozella JC, Stern PJ, Murdock PA. The fate of failed bone graft surgery for scaphoid nonunions. J Hand Surg [Am] 1989;14:800-6.

22. Boyer MI, von Schroeder HP, Axelrod TS. Scaphoid nonunion with avascular necrosis of the proximal pole: treatment with a vascularized bone graft from the dorsum of the distal radius. J Hand Surg [Br] 1998;23:686-90.

23. Cooney WP, Linscheid RL, Dobyns JH, Wood MB. Scaphoid nonunion: role of anterior interpositional bone grafts. J Hand Surg [Am] 1988;13:635-50.

24. Malizos KN, Dailiana ZH, Kirou M, et al. Longstanding nonunions of scaphoid fractures with bone loss: successful reconstruction with vascularized bone grafts. $J$ Hand Surg [Br] 2001;26:330-4

25. Straw RG, Davis TR, Dias JJ. Scaphoid nonunion: treatment with a pedicled vascularized bone graft based on the 1,2 intercompartmental supraretinacular branch of the radial artery. J Hand Surg [Br] 2002;27:413.

26. Dell PC, Burchardt H, Glowczewski FP Jr. A roentgenographic, biomechanical, and histological evaluation of vascularized and non-vascularized segmental fibular canine autografts. J Bone Joint Surg [Am] 1985;67-A:105-12.

27. Chacha PB. Vascularised pedicular bone grafts. Int Orthop 1984;8:117-38.

28. Mathoulin C, Haerle M. Vascularized bone graft from the palmar carpal artery for treatment of scaphoid nonunion. J Hand Surg [Br] 1998;23:318-23.

29. Mathoulin C, Brunelli F. Further experience with the index metacarpal vascularized bone graft. J Hand Surg [Br] 1998;23:311-17.

30. Kawai H, Yamamoto K. Pronator quadratus pedicled bone graft for old scaphoid fractures. J Bone Joint Surg [Br] 1988;70-B:829-31.

31. Guimberteau JC, Panconi B. Recalcitrant non-union of the scaphoid treated with a vascularized bone graft based on the ulnar artery. J Bone Joint Surg [Am] 1990;72A:88-97.

32. Zaidemberg C, Siebert JW, Angrigiani C. A new vascularized bone graft for scaphoid nonunion. J Hand Surg [Am] 1991;16:474-8.

33. Tambe AD, Cutler L, Stilwell J, et al. Scaphoid non-union: the role of vascularized grafting in recalcitrant non-unions of the scaphoid. J Hand Surg [Br] 2006;31:185-90.

34. Hara T, Horii E, An KN, et al. Force distribution across wrist joint: application of pressure-sensitive conductive rubber. J Hand Surg [Am] 1992;17:359-47.

35. Schnitzler CM, Biddulph SL, Mesquita JM, Gear KA. Bone structure and turnover in the distal radius and iliac crest: a histomorphometric study. J Bone Miner Res 1996;11:1761-8

36. Vender MI, Watson HK, Wiener BD, Black DM. Degenerative change in symptomatic scaphoid nonunion. J Hand Surg [Am] 1987;12:514-19.

37. Steinmann SP, Bishop AT, Berger RA. Use of the 1, 2 intercompartmental supraretinacular artery as a vascularized pedicle bone graft for difficult scaphoid nonunion. J Hand Surg [Am] 2002;27:391-401.

38. Ramamurthy C, Cutler L, Nuttall D, et al. The factors affecting outcome after nonvascular bone grafting and internal fixation for nonunion of the scaphoid. J Bone Joint Surg [Br] 2007;89-B:627-32

39. Merrell GA, Wolfe SW, Slade JF III. Treatment of scaphoid nonunions: quantitative meta-analysis of the literature. J Hand Surg [Am] 2002;27:685-91. 